\title{
Low energy shock response of a melt cast simulant material
}

\author{
David Drouet ${ }^{1,2}$, Patrice Bailly ${ }^{2}$, Julien Pavier $^{1}$, Nicolas Eches ${ }^{1}$ and Jean-Luc Hanus ${ }^{2}$ \\ ${ }^{1}$ NEXTER Munitions, 7 route de Guerry, 18000 Bourges, France \\ ${ }^{2}$ INSA CVL, Univ. Orléans, Univ. Tours, LaMé EA7494, 88 Boulevard Lahitolle F-18022 Bourges, France
}

\begin{abstract}
To manufacture its insensitive munitions (MURAT MUnitions à Risque ATténué), NEXTER Munitions uses a melt cast explosives as an EIDS (Extremely Insensitive Detonating Substance). Unlike commonly used and well-documented EIDSs such as PBX, melt cast have a high volumetric matrix percentage. Moreover, in its life cycle, the ammunition can undergo severe loads, such as cannon firing, accidental shocks and terminal ballistics events. The objective of this paper is therefore to analyse, how these dynamic loads induce changes in the material (damage, cracking, de-cohesion), and then, to evaluate how these alterations influence the pyrotechnic properties of the melt cast explosive. To address the first point, we delimited the scope of the study in pressure and strain rate ranges which corresponds to the context of the ammunition. To safely explore this area, we have created an inert material that is morphologically and mechanically representative of the melt case explosive. It is used to setup and validate the experimental technic that will be applied to damage the melt cast explosive in the future. In this article the mechanical behaviour of the inert material is investigated under simple compression and passive confinement. This was done under the quasi-static and dynamic regimes thanks to a compression press and a Split Hopkinson Pressure Bars setup. Firstly, the results obtained show the emergence of a damage which increases with the loss of cohesion of the material during the test. This seems to be related to the extension strain. Then, for all tests, a strain rate dependent mechanical response is observed. Finally, the end of the test shows the material behaviour without cohesion. Then, a rate dependent ultimate shear criterion is deduced. To complete the interpretation these results, a model is proposed. It intends to be simple as it tries to describe the whole degradation of the material with a unique scalar parameter.
\end{abstract}

\section{Introduction}

In the field of ammunition, the explosive is subjected to a wide range of mechanical interactions, from low strain rates and pressures during the handling of munitions, to high pressure and strain rates loadings during the firing or terminal ballistics. In all these circumstances, the ammunition producer must ensure the safety and performance of its products. This involves understanding the damage mechanisms in the melt cast explosive and the consequences on its pyrotechnic safety and performances. However, despite an abundant presence in large calibre ammunition, this family of explosives remains poorly documented. Unlike the more studied polymer bounded explosives (PBX) or compressed explosives (TATB, HMX), the melt cast explosives have a matrix percentage that can exceed $40 \%$ vol. This implies a different sensitivity of the pyrotechnic chain to the mechanical damage. In this context, it seems to be important to study the low energy shock behaviour of the insensitive melt cast explosives. Before operating with a real explosive, an inert simulant is designed. It is used to develop an experimental set up and a post-processing procedure under safe conditions and at reduced costs.
Thus, this article mainly focuses on the experimental procedure used with this inert simulant and the tests results obtained. In the last part we choose to pay attention to the manifestation of damage specific to the melt cast materials which is analysed with a single damage parameter model.

\section{Experimental set-up}

\subsection{Inert sample preparation}

In order to set up the confined compression procedure with the XF-11585, we used a simulant material designed to be morphologically and mechanically representative of the melt cast explosive. It is important to notice that the inert simulant comes from a laboratory procedure with less reproducibility than its active counterpart. A segregation porosity and cooling cracks may appear which influence the mechanical behaviour by weakening the shear strength of the material. In order to rule out these phenomena, a water bath is used during the material manufacturing process. However, vapours of this bath may contaminate the material during the casting and change in turn the strength and stiffness of 
the material. As a consequence, using a concrete material analogy, a curing time of one month was applied to regain mechanical properties.

\subsection{Passive confinement cell}

The passive containment is made with brass rings of chosen thicknesses. That allows us to cover the desired pressure range from 30 to $150 \mathrm{MPa}$ that is expected to occur during an artillery shell life [5]. The sample, coated with an epoxy resin, is put into the ring. The resin fills up any clearance between the test material and the ring. Without this filling material the inert and its active counterpart will break before the beginning of the confinement. The resulting assembly is described in Fig. 1. Due to the elastoplastic response of the passive confinement, two different stages are expected in the mechanical response of the tested material. During the first stage, elastic response of the confinement, there will be a strong increase in pressure and the shear will remain low. While, in the second stage, perfect plastic response of the ring, the confinement pressure will be maintained and the shear will increase up to a critical state in the damaged material.

Due to the rotational symmetry on the axis of the ring (Fig. 1), the expression of the stress and strain tensors are simplified (eq. 1 and 2).

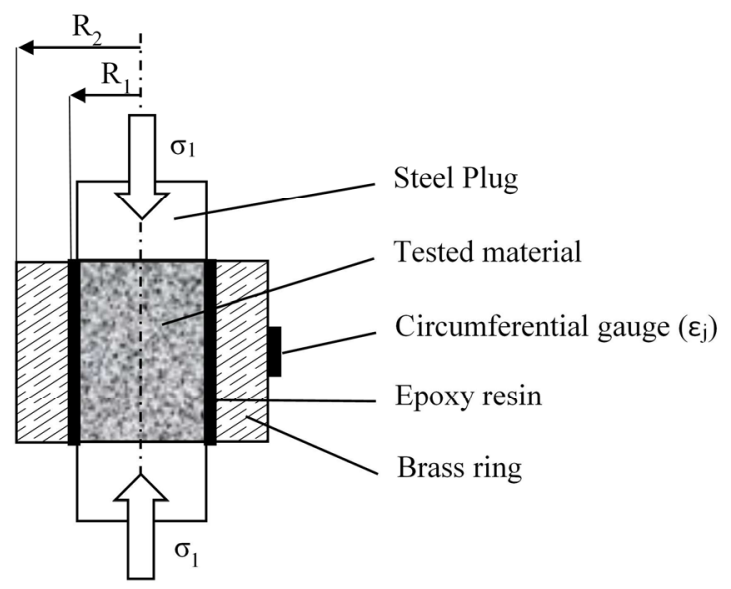

Fig. 1. 2D section of the confined compression assembly.

$$
\begin{aligned}
& \underline{\underline{\sigma}}=\left(\begin{array}{ccc}
\sigma_{1} & 0 & 0 \\
0 & \sigma_{2} & 0 \\
0 & 0 & \sigma_{2}
\end{array}\right) \\
& \underline{\underline{\varepsilon}}=\left(\begin{array}{ccc}
\varepsilon_{1} & 0 & 0 \\
0 & \varepsilon_{2} & 0 \\
0 & 0 & \varepsilon_{2}
\end{array}\right)
\end{aligned}
$$

We can deduce the state of the loads in the confinement with $\varepsilon_{1}, \varepsilon_{2}, \sigma_{1}$ and $\sigma_{2}$. The values for the longitudinal strain $\left(\varepsilon_{1}\right)$ and stress $\left(\sigma_{1}\right)$ are given by the axial displacement of the plugs and the force sensor of the press or the post-processing of the strain gauges of the Split Hopkinson Pressure Bars. The circumferential strain $\left(\varepsilon_{\mathrm{j}}\right)$ is measured by a gauge on the ring surface. Thanks to a known rate independent, elastic perfectly plastic mechanical behaviour of the brass [4]. We use its young modulus $\mathrm{E}_{\mathrm{l}}$, Poisson ratio $\mathrm{v}_{\mathrm{l}}$, and yield stress $\sigma_{\mathrm{e}}$, to get the circumferential stress $\left(\sigma_{2}\right)$ and strain $\left(\varepsilon_{2}\right)$ from an analytical calculation.

During the first stage, the ring behaves elastically and we have:

$$
\begin{array}{r}
\varepsilon_{2}=\frac{\left(1+v_{l}\right) R_{2}^{2}+\left(1-v_{l}\right) R_{1}^{2}}{2 R_{1}^{2}} \\
\sigma_{2}=\left(\frac{R_{2}^{2}-R_{1}^{2}}{2 R_{1}^{2}}\right) E_{l} \varepsilon_{j}
\end{array}
$$

Then, during the transition between elastic and plastic behaviour, which starts at $\varepsilon_{j}>\frac{\sigma_{e} R_{1}^{2}}{E_{l} R_{2}^{2}}$, we use:

$$
\begin{array}{r}
\varepsilon_{2}=\frac{\sigma_{e}\left(1-v_{l}\right)}{E_{l}}\left(\ln \left(\frac{R_{1}}{R_{2}} \sqrt{\frac{\sigma_{e}}{E_{l} \varepsilon_{j}}}\right)+\frac{1}{2 R_{2}^{2}}+\frac{E_{l}^{2} R_{2}^{2} \varepsilon_{j}^{2}}{\sigma_{e} R_{1}^{2}}\right) \\
\sigma_{2}=\sigma_{e}\left(\frac{\sigma_{e}-E_{l} \varepsilon_{j}}{2 \sigma_{e}}+\ln \left(\frac{R_{2}}{R_{1}} \sqrt{\frac{E_{l} \varepsilon_{j}}{\sigma_{e}}}\right)\right)
\end{array}
$$

Finally, the ring is fully plasticized when $\varepsilon_{j}>\frac{\sigma_{e}}{E_{l}}$. So, we use:

$$
\begin{gathered}
\varepsilon_{2}=\left(\frac{R_{2}^{2}}{R_{1}^{2}}-\left(1-v_{l}\right) \ln \left(\frac{R_{2}}{R_{1}}\right)\right) \varepsilon_{j} \\
\sigma_{2}=\sigma_{e} \ln \left(\frac{R_{2}}{R_{1}}\right)
\end{gathered}
$$

Five different ring thicknesses allow us to cover the pressure domain from 30 to $150 \mathrm{MPa}$.

Knowing $\varepsilon_{1}, \varepsilon_{2}, \sigma_{1}$ and $\sigma_{2}$ in the material, we can characterize the mechanical response. To depict the pressure and shear behaviours of the material, the mechanical response is expressed as a function of two invariants. The pressure and the Von Mises equivalent stress are selected for the stress tensor:

$$
\begin{gathered}
P=\frac{\operatorname{tr}(\underline{\underline{\sigma}})}{3}=\frac{\sigma_{1}+2 \sigma_{2}}{3} \\
\underline{\underline{s}}=\underline{\underline{\sigma}}+P \underline{\underline{1}} \\
\sigma_{e q}=\sqrt{\frac{3 \underline{\underline{s}: \underline{\underline{S}}}}{2}}=\sigma_{1}-\sigma_{2}
\end{gathered}
$$

And their respective dual values for the strains, the volumetric strain $\theta$ and the Von Mises equivalent strain $\varepsilon_{\text {eq }}$ :

$$
\begin{gathered}
\theta=\operatorname{tr}(\underline{\underline{\varepsilon}})=\varepsilon_{1}+2 \varepsilon_{2} \\
\underline{\underline{e}}=\underline{\underline{\varepsilon}}+\frac{\theta}{3} \underline{=} \\
\varepsilon_{e q}=\sqrt{\frac{2 \underline{\underline{e} \underline{\underline{e}}}}{3}}=2 \frac{\varepsilon_{1}-\varepsilon_{2}}{3}
\end{gathered}
$$

\subsection{Quasi-static experiments}


Uniaxial passive confined compression tests are performed with an MTS press. These quasi-static experiments give us an overview of the material behaviour at low strain rates under monotonic or unloading-reloading cycling. They are also used to size the dynamic solicitations of our SHPB campaign. Furthermore, by using biaxial strain gages glued on the ring, we carried out additional tests that made it possible to quantify the friction induced by the addition of the resin. This effect is shown to be negligible, in comparison to dispersion due to measurement noise and scattering in material shear strength.

\subsection{Dynamic SHPB experiments}

To perform the high strain rate tests, a Split Hopkinson Pressure Bar's device is used. It is composed of three elements with a diameter of $20 \mathrm{~mm}$ (Fig. 2). The impactor is $1.25 \mathrm{~m}$ in length, the input bar is $3 \mathrm{~m}$ in length and the output bar is $1.8 \mathrm{~m}$ in length. They are made of steel with a low young modulus of $160 \mathrm{GPa}$ and a density of $7800 \mathrm{~kg} / \mathrm{m}^{3}$.

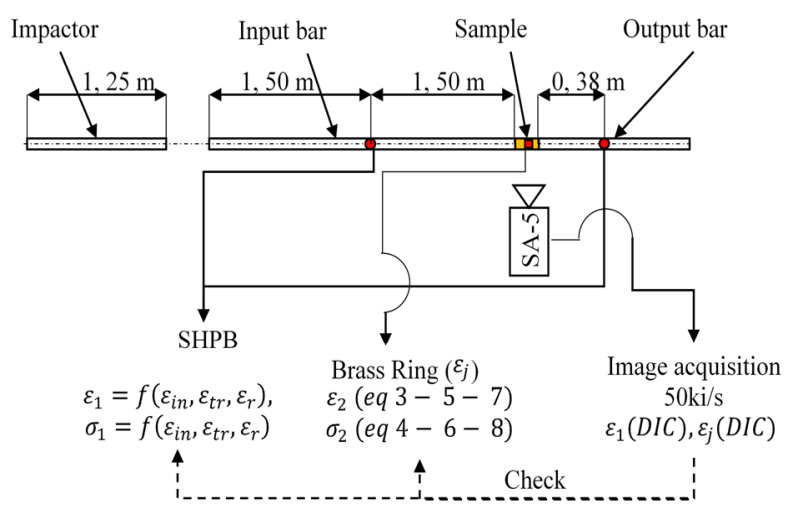

Fig. 2. Split Hopkinson Pressure Bar's measuring chain.

In addition to the classical strain gauges measurements, a very high speed camera (Photron SA-5) is used to both track the displacements at the sample/bar interfaces and to get full field measurements at the ring surface with the Digital Image Correlation technique (Correli Q4 software [7]). These redundant values are mainly used to readjust, if appropriate, the time-shifting between strain recordings from gauges attached to the bars and to the ring. A delay in response might appear due to a mechanical looseness between bars and plugs or/and between plugs and ring which could lead to a wrong evaluation of the mechanical state in the specimen.

\section{Results and discussion}

Compression tests with five confinement levels are performed. The mechanical response is shown in the hydrostatic stress / volumetric strain plane (Fig. 3) and in the Von Mises equivalent stress / strain plane (Fig. 4).

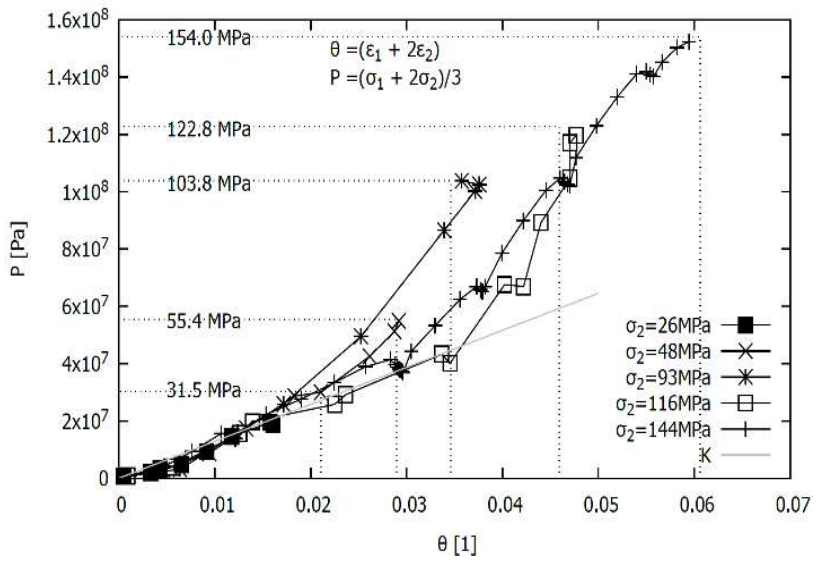

Fig. 3. Hydrostatic stress $(\mathrm{P})$ as a function of volumetric strain $(\theta)$ for five levels of confinement $\left(\sigma_{2}\right)$.

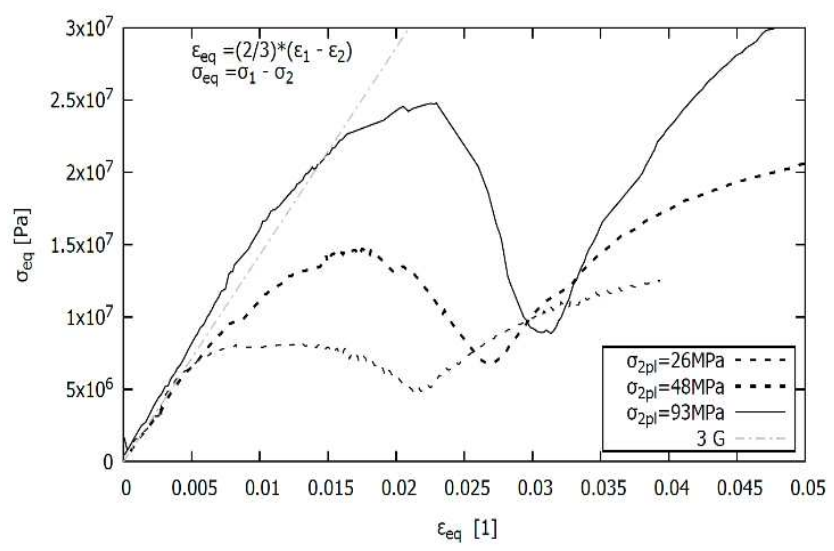

Fig. 4. Von-Mises equivalent stress $\left(\sigma_{\text {eq }}\right)$ as a function of the Von-Mises equivalent strain $\left(\varepsilon_{\mathrm{eq}}\right)$ for three confinement levels.

With those measurements we are able to draw the loading path encountered by the material during the test (Fig. 5). This allows us to identify different stages during the confined compression test:

- Pre-confinement,

- Stage 1: Linear elastic behaviour (undamaged),

- Stage 2: Progressive material damage,

- Stage 3: Fully plasticized brass ring.

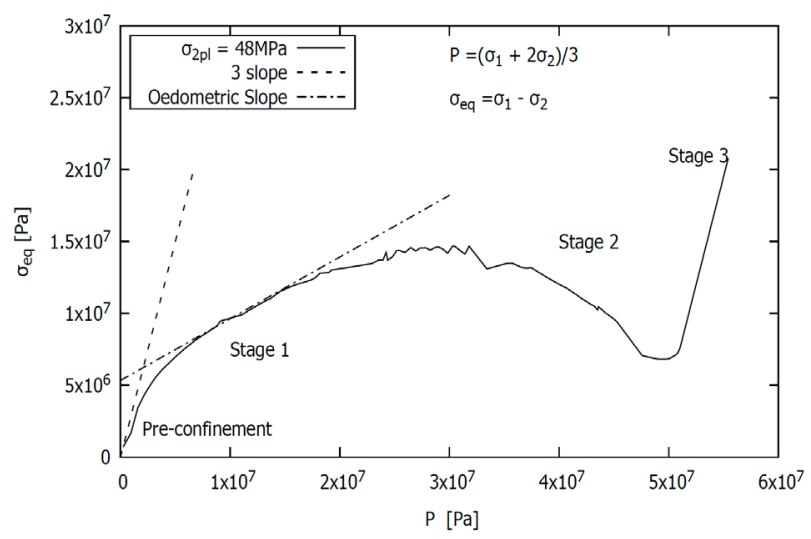

Fig. 5. Loading path in the stress plane $\left(\sigma_{e q}, P\right)$ (confinement of $26 \mathrm{MPa}$ ). 
The pre-confinement phase is a phase with a very short duration which appears at the beginning of the test (Fig. 5). The confinement pressure is properly evaluated but the sample radial deformation $\varepsilon_{2}$ is under evaluated with the values obtained from the ring. This is mainly due to the response of the filling material that absorbs a small amount of strain before the complete effectiveness of the confinement. We compensate this effect by assuming a material elastic response that corrects the values of $\varepsilon_{2}$.

\subsection{Linear elastic behaviour (Stage 1)}

During the confined compression, a linear behaviour is observed. It appears after the pre-confinement phase and before the Stage 2. In this short stage, the measurements are consistent with that of an elastic material. The values of the different elastic moduli identified are given in Table. 1 for both quasi-static and dynamic experiments. At this stage, it must be emphasized that the values identified during dynamics experiments are only apparent moduli since they may reflect a viscoelastic behaviour.

Table 1. Elastic moduli identified during elastic behaviour.

\begin{tabular}{|c|c|c|c|}
\hline & $\begin{array}{c}\mathrm{QS} \\
\left(1,85 \mathrm{E}^{-4} \mathrm{~s}^{-1}\right)\end{array}$ & & $\begin{array}{c}\text { Dyn } \\
\left(300-600 \mathrm{~s}^{-1}\right)\end{array}$ \\
\hline $\mathrm{K}(\mathrm{GPa})$ & $1,2+/-0,45$ & $\mathrm{~K}_{\text {app }}(\mathrm{GPa})$ & $4,2+/-1,5$ \\
\hline $\mathrm{G}(\mathrm{GPa})$ & $0,4+/-0,16$ & $\mathrm{G}_{\text {app }}(\mathrm{GPa})$ & $1,3+/-0,44$ \\
\hline v & $0,33+/-0,04$ & $\mathrm{v}_{\mathrm{app}}$ & $0,35+/-0,02$ \\
\hline $\mathrm{E}(\mathrm{GPa})$ & $1,2+/-0,42$ & $\mathrm{E}_{\text {app }}(\mathrm{GPa})$ & $3,7+/-1,2$ \\
\hline
\end{tabular}

This assumption was supported by a complementary analysis performed with a DMA press. A moderate stimulation amplitude, with a frequency ranging from 1 to $200 \mathrm{~Hz}$, is applied to the specimen. This gives a strain rate between $6 \mathrm{E}^{-4} \mathrm{~s}^{-1}$ to $0,1 \mathrm{~s}^{-1}$. From the dynamic test (Table. 1) we take as a first approximation that, the Poisson coefficient can be considered as constant. Then, we used a Kelvin-Voigt model to extract the elastic viscosity $\eta=57200 \mathrm{~Pa}$. s. These viscoelastic parameters are used in the last part of this paper to describe the viscoelastic part of a viscoelastic-viscodamage model (Section 4).

\subsection{Progressive Damage (Stage 2)}

During this phase of the test the material response in shear is modified. The occurrence of damage, highlighted in the Von Mises Stress/Von Mises Strain plane (Fig. 4), appears to be dependent on the level of confinement. As a consequence, a good driving variable for the damage might be the extension strain ( $\varepsilon_{2}$ for us) as it is minimized by higher confinements.

To gain a more precise idea of the changes in the material response, we also performed relaxation, unloading-reloading cycles and tests at different strain rates thanks to the Split Hopkinson Pressure Bars.
- Unloading-reloading cycles: With the cycling of the material we were able to evaluate the amount of permanent strain in volumetric and equivalent Von Mises strains. We observed an apparent stiffening of the bulk modulus while the shear modulus seems to be unchanged.

- Strain rate sensitivity: On Fig. 6, we have the same confinement used with two different strain rates. The SHPB tests show a major influence of the strain rate on the Von Mises equivalent stress and Pressure thus revealing the viscoelasticy and the rate dependence of damage in the material response.

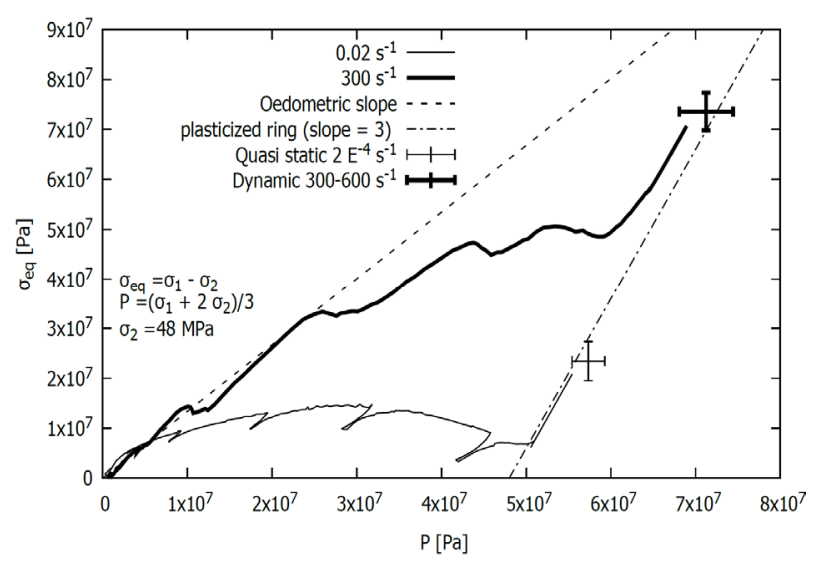

Fig. 6. Effect of strain rate on the loading path (confinement of $48 \mathrm{MPa}$ ) confinement ring: top curve $\left(300 \mathrm{~s}^{-1}\right)$ from a SHPB test, bottom curve from a relaxation test with 30 s pauses.

\subsection{Damaged material (Stage 3)}

At the end of the test, the brass ring is fully plasticized, which imposes the loading path to the material by forcing it to follow a line of slope 3 up to its critical shear state. In Fig.5, we identified this behaviour in Stage 3. We collected all the endpoints of our tests and merge them in Fig.7. Thus, we were able to see that the material exhibits a straight failure envelope. We can also notice a strong strain rate dependence of the criterion.

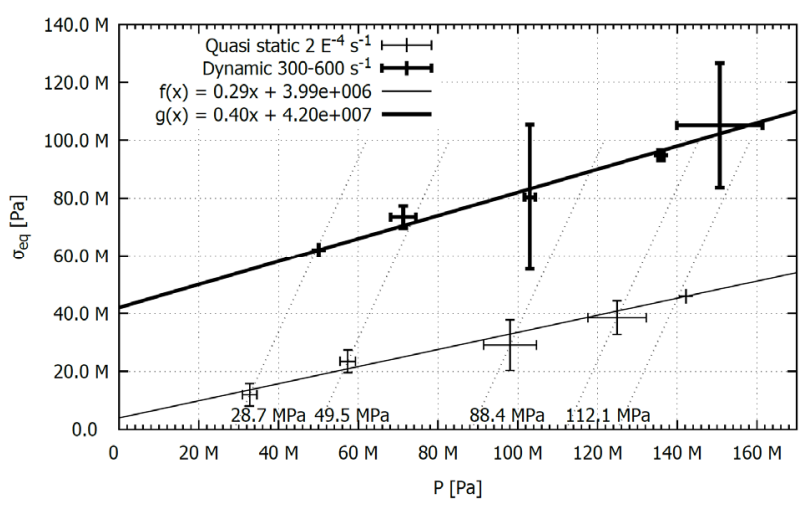

Fig. 7. Critical shear state in dynamic and quasi static experiments in the Pressure/Von Mises stress plane. The two linear behaviour demonstrate a correlation coefficient of 0.98 for $\mathrm{g}(\mathrm{x})$ and 0.97 for $\mathrm{f}(\mathrm{x})$. 


\section{Modelling: scalar model}

In the previous part, we have spotted the damage that occurs in the material during uniaxial confined compression experiments. This damage, as well as initial morphological phenomena (porosity, crack, segregation), is known to influence the pyrotechnics properties [1-2]. In a first modelling attempt, we will pay attention to the matrix damaging. As a consequence some other evidences will be neglected:

- Stiffening of the bulk modulus,

- Permanent volumetric and Von-Mises equivalent strains,

- Behaviour of the damaged material with the plastic brass ring (Stage 3).

The final goal of this modelling is to be able to quantify, in a volume of explosive, the value of damage in the matrix. With this knowledge, we wish to improve the prediction of the shockwave propagation in the energetic material and its possible transition from the detonation to deflagration.

This paper is focused on the examination of a simple scalar damage model. The evolution of the damage internal variable is calculated from the strains $\varepsilon_{1}(\mathrm{t}), \varepsilon_{2}(\mathrm{t})$ with an explicit algorithm:

1. The equations (12) and (14) provide $\varepsilon_{\mathrm{eq}}$ and $\theta$.

2. The threshold function is expressed as [3]:

$$
\begin{gathered}
F\left(\varepsilon^{+}, D\right)=\operatorname{aA}\left(\operatorname{atan}\left(\frac{\varepsilon^{+}}{a}\right)-\operatorname{atan}\left(\frac{K_{0}}{a}\right)\right)-D \\
\text { where } \varepsilon^{+}=\max \left(\left\langle\varepsilon_{i}\right\rangle\right)
\end{gathered}
$$

With $\kappa_{0}$, the threshold strain, a and A, two material dimensionless parameters.

3. The strain rate damage dependence is linked to the threshold function value [6]:

$$
\dot{D}=D_{\infty}\left(1-e^{-c\left(F\left(\varepsilon^{+}, D\right)\right)}\right)
$$

4. Then, the damage is applied to the shear modulus:

$$
G=G_{0}(1-D)+D G_{1}
$$

5. That allows us to calculate the Von Mises equivalent stress (Fig.8) :

$$
\sigma_{e q}=3 G \varepsilon_{e q}+\frac{3 \eta}{2(1+v)} \dot{\varepsilon}_{e q}
$$

6. Then the pressure response of the material is expressed as follows (Fig.9)

$$
P=\frac{1}{3(1-2 v)}(E \theta+\eta \dot{\theta})+K_{2} \theta^{2}
$$

We are able to draw the loading path (Fig.10).
7. Finally, we check if the model predictions are valid by comparing the calculated axial and radial stresses against the experimental ones (Fig. 11 and Fig. 12):

$$
\begin{aligned}
& \sigma_{1}=P+\frac{2 \sigma_{e q}}{3} \\
& \sigma_{2}=P-\frac{\sigma_{e q}}{3}
\end{aligned}
$$

In the following curves (Fig. 8 to Fig. 12), we used the same set material parameters for quasi static $\left(2 \mathrm{E}^{-4} \mathrm{~s}^{-1}\right)$ and dynamic $\left(300 \mathrm{~s}^{-1}\right)$ experiments (Table. 2), In these experiments the ring thickness generates a confinement pressure equal to $144 \mathrm{MPa}$.

Table 2. Scalar Model parameters.

\begin{tabular}{|c|c|c|c|}
\hline Parameters & Values & Parameters & Values \\
\hline G0 (MPa) & 800 & $\mathbf{K 2} \mathbf{( P a )}$ & $4,5 \mathrm{E}^{10}$ \\
\hline $\mathbf{G 1}(\mathbf{M P a})$ & 20 & $\boldsymbol{\eta} \mathbf{( k P a )}$ & 59,5 \\
\hline $\mathbf{v}$ & 0,38 & $\mathbf{a}$ & 0,015 \\
\hline $\mathbf{E}(\mathbf{G P a})$ & 1.2 & $\mathbf{A}$ & 83 \\
\hline $\mathbf{D}_{\boldsymbol{\infty}}$ & 5200 & $\kappa_{\mathbf{0}}$ & 0,0019 \\
\hline $\mathbf{c}$ & 9000 & \multicolumn{2}{l}{} \\
\cline { 2 - 4 } & \multicolumn{3}{|r}{}
\end{tabular}

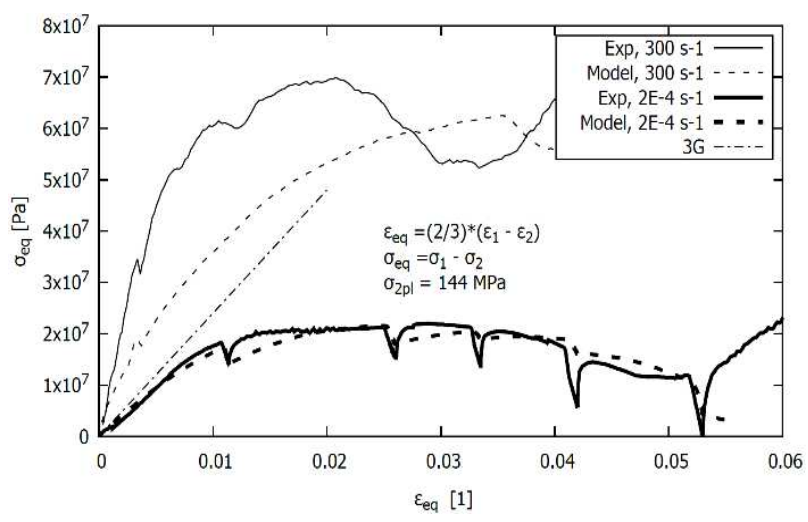

Fig. 8. Behaviour of the model in the Von Mises stress / Von Mises strain plane.

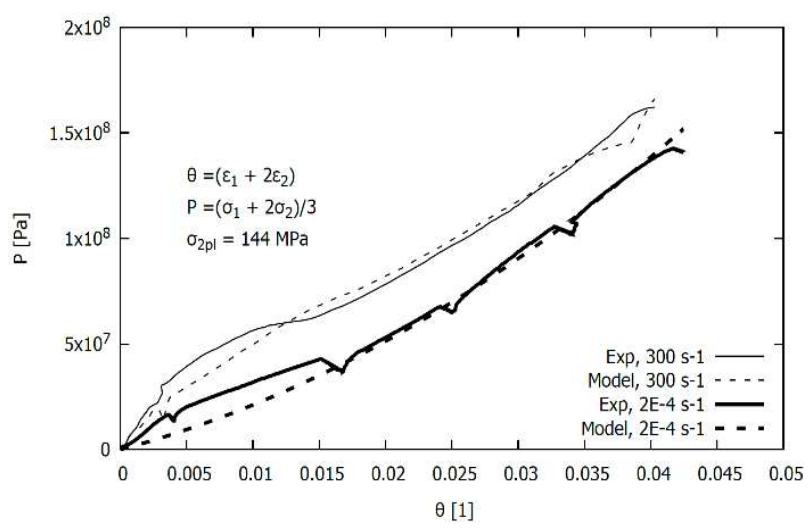

Fig. 9. Behaviour of the model in the pressure / volumetric strain plane. 


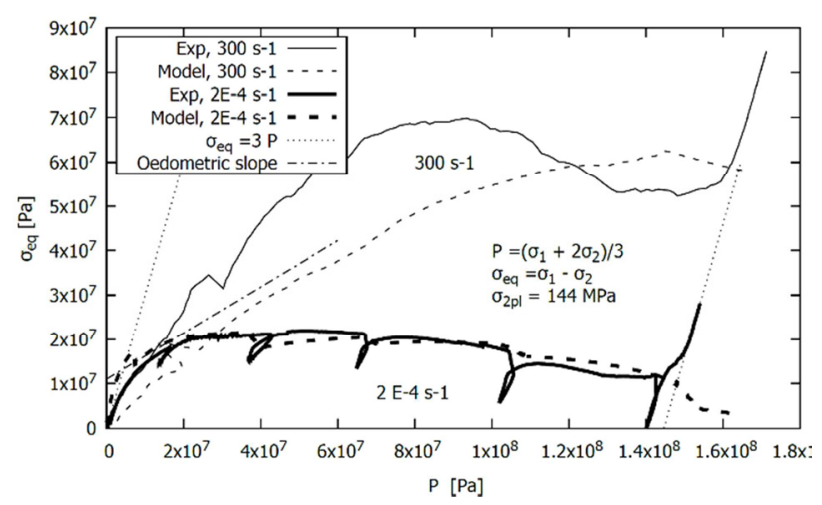

Fig. 10. Response of the model along the loading path.

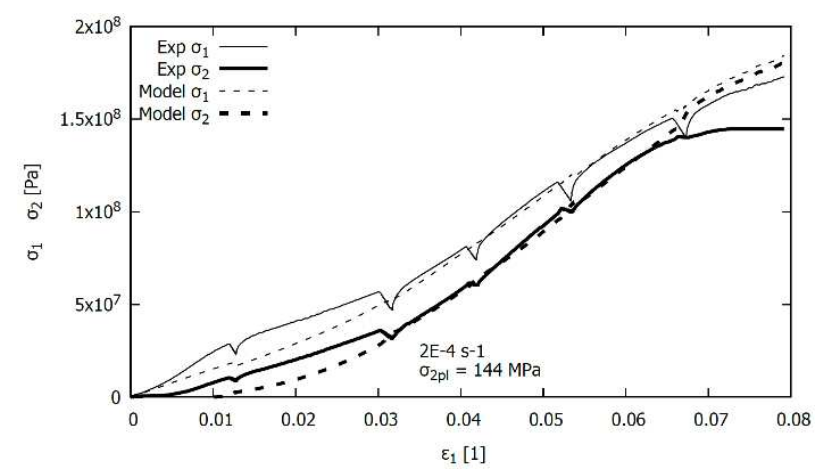

Fig. 11. Low strain rate response of the model compared to the experimental results.

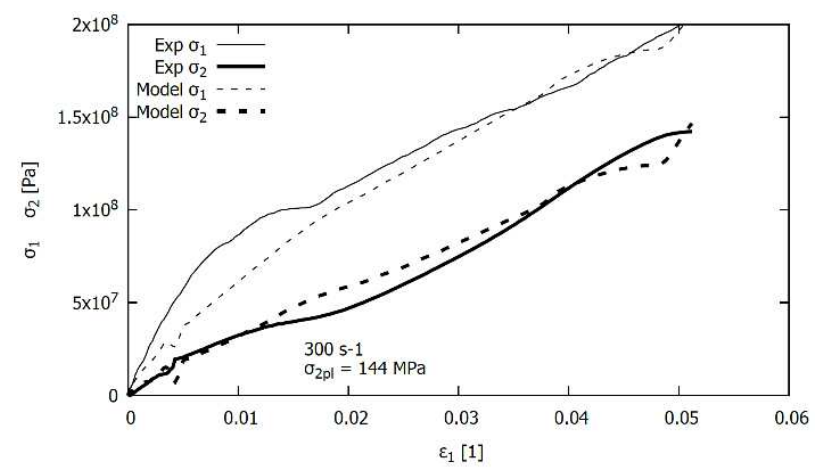

Fig. 12. High strain rate response of the model compared to the experimental results.

As we can see on all these graphics, this very simple model manages to get close to the experiments. By considering Fig. 8 and Fig. 10, we can notice that there is insufficient rate sensitivity in the model to reproduce the shear response at high speed. We need to thoroughly analyse our dynamics experiments results and to optimize our set of material parameters only roughly evaluated in this paper.

\section{Conclusion}

In this paper we presented the development of an experimental setup to characterize the mechanical response of melt-cast explosives submitted to high pressure confinement and a wide spectrum of strain rates. Thanks to an inert simulant, morphologically and mechanically equivalent, this protocol was defined under safe conditions. Moreover, the obtained results allow us to describe a behaviour representative of theses explosives. Thus, selecting the matrix damage phenomenon we modelled the damage processing of that type of material with a single scalar damage model in a satisfactory manner. We expect to apply this approach to the actual melt cast explosives (XF-11585) and to improve the material model by introducing permanent strains and stiffening of the material's moduli.

We thank Stephane Meo and Mathieu Venin from the CERMEL for their assistance with the DMA analysis.

\section{References}

1. E.L. Baker, Use of laboratory setback activator tests to asses suitability for gun launch. Orr, MSIAC (2017)

2. G. Baudin, Mémoire d'habilitation à diriger des recherches, Physique des explosifs : de l'amorçage aux effets des explosifs condensés, Science des matériaux, Université Paris Ouest Nanterre La Défense (2013)

3. M.Chambart, Phd : Endommagement anisotrope et comportement dynamique des structures en béton armé jusqu'à la ruine, ENS-Cachan (2009)

4. P. Bailly, F. Delvare, J. Vial, J.L. Hanus, M. Biessy, D. Picart. Dynamic behavior of an aggregate at simultaneous high pressure and strain rate: SHPB triaxial tests, International Journal Of Impact Engineering (2010)

5. R.W. Collett, Measurement of in-bore setback pressure on projectile warheads using hard-wire telemetry. International Telemetering Conference Proceedings. (1983)

6. O.Allix, J.F. Deü, Delayed-damage modelling for fracture prediction of laminated composites under dynamic loading. Engng Trans E 45, 1, p 29-46 (1997)

7. Hild F, Roux S Correli Q4 a software for "finiteelement" displacement field measurements by digital image correlation. Tech. rep. , LMT Cachan (2008) 